Rubrikherausgeber:

Tom Schaberg, Rotenburg (Koordination)

Roland Buhl, Mainz

Adrian Gillissen, Leipzig
Karl Häußinger, Gauting

Rolf Merget, Bochum

Claus Vogelmeier, Marburg

T. O. F. Wagner, Frankfurt
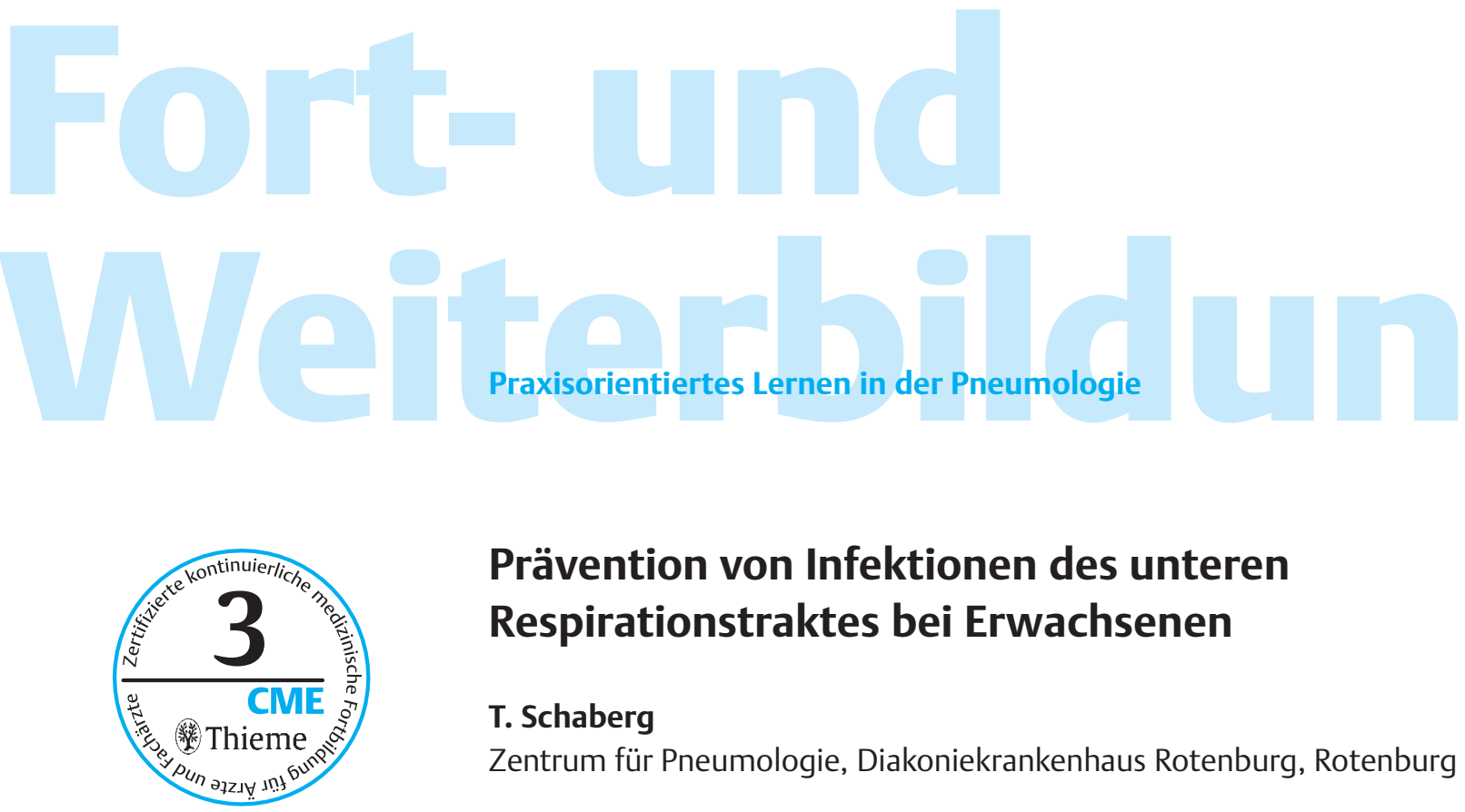

\title{
Prävention von Infektionen des unteren Respirationstraktes bei Erwachsenen
}

\section{T. Schaberg}

Zentrum für Pneumologie, Diakoniekrankenhaus Rotenburg, Rotenburg

\section{Weiterbildungsziele}

In diesem Beitrag sollen folgende Weiterbildungsziele vermittelt werden:

1. Influenza-Schutzimpfung

2. Medikamentöse Prävention der Influenza-Infektion

3. Pneumokokken-Schutzimpfung

4. Antibiotika zur Prophylaxe bei chronischer Bronchitis und COPD

5. Prävention von Infektionen des tiefen Respirationstraktes durch die frühzeitige antibiotische Therapie von Infekten des oberen Respirationstraktes

6. Langwirksame Beta-2-Mimetika, Vagolytika und inhalative Steroide als präventive Maßnahme gegenüber tiefen Atemwegsinfektionen bei Patienten mit COPD
7. Prävention tiefer Atemwegsinfekte durch regelmäßige Physiotherapie

8. Orale Mukolytika zur Prävention tiefer Atemwegsinfektionen

9. Homöopathische Substanzen als präventive Strategie gegenüber tiefen Atemwegsinfektionen

10. Orale Immunisationsstrategien mit Bakterienextrakten

11. Fazit für die Praxis 
Infektionen des unteren Respirationstraktes sind sehr häufig.

Die Vakzine wird intramuskulär im Herbst appliziert.

Eine jährliche Wiederholung der Impfung ist wegen der sich ständig ändernden antigenen Eigenschaften der Influenzaviren notwendig.
Infektionen des unteren Respirationstraktes sind sehr häufig. Neben der akuten Bronchitis, die in der Regel durch Viren bedingt ist und vermutlich 10-20\% der Bevölkerung einmal jährlich trifft, spielen vor allen Dingen infektionsbedingte Exazerbationen der chronischen Bronchitis und der COPD sowie ambulant erworbene Pneumonien eine große Rolle. Bei einer geschätzten Prävalenz der chronischen Bronchitis und COPD in der Bevölkerung von 5\% kann von mehreren Millionen infektionsbedingter Exazerbationen dieser Krankheitsbilder pro Jahr ausgegangen werden. An einer ambulant erworbenen Pneumonie erkranken jährlich ca. 800000 Personen in Deutschland.

Im Folgenden sollen Möglichkeiten der Prävention bzw. Prophylaxe von Infektionen des unteren Respirationstraktes dargestellt werden und ihre Effektivität anhand der zur Zeit verfügbaren Literatur bewertet werden.

\section{Influenza-Schutzimpfung}

Der zur Zeit weltweit am häufigsten eingesetzte Impfstoff ist eine inaktivierte Spaltvakzine, die jeweils im Frühjahr nach Empfehlungen einer Expertenkommission der Weltgesundheitsorganisation und des Center of Disease Control and Prevention [1] der erwartbaren aktuellen epidemiologischen Situation im kommenden Herbst, Winter und Frühjahr angepasst wird [2]. Diese Vakzine wird intramuskulär im Herbst appliziert. Eine jährliche Wiederholung der Impfung ist wegen der sich ständig ändernden antigenen Eigenschaften der Influenzaviren (Abb. 1 u. 2) notwendig [3].

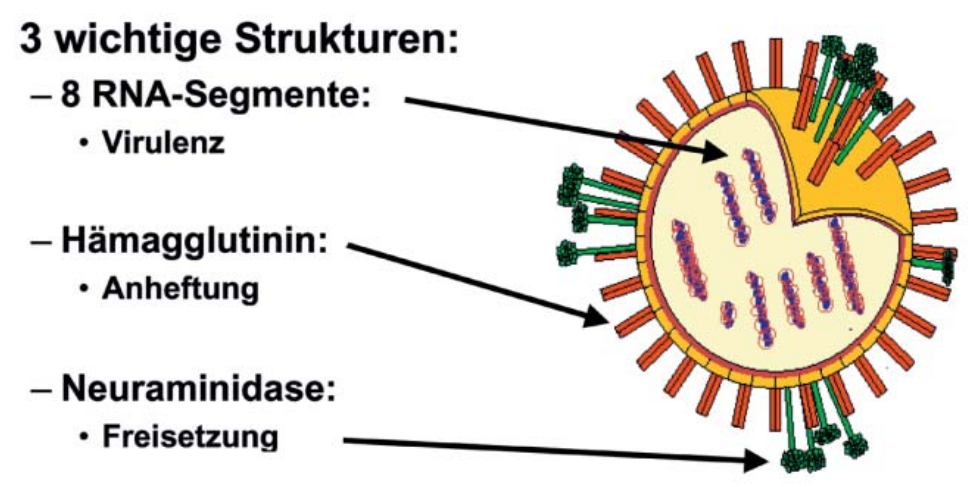

Abb. 1 Influenza-Viren (nach Nicholson KG, Wood JM, Zambon M. Influenza. Lancet 2003; 362: 1733 - 1745).

Als Kontraindikation kann nur die Unverträglichkeit gegenüber Hühnereiweiß aufgeführt werden, da die Spaltvakzine in Hühner-Embryonen produziert wird. Zukünftig können auch Spaltvakzine in Zellkulturen produziert werden, hier entfällt dann vermutlich diese Kontraindikation. Systemische Nebenwirkungen treten nach der Anwendung der Spaltvakzine nur in der Größenordnung des Plazeboeffektes auf. Lokale Reaktionen werden jedoch von 10-20\% der Geimpften beschrieben und umfassen überwiegend kurzfristige Schmerzen im Bereich der Impfstelle. Es ist umstritten, ob bei der Impfung von Patienten mit einem instabilen Asthma bronchiale oder einer fortgeschrittenen COPD Exazerbationen der Grunderkrankung induziert werden können. Wahrscheinlich ist dies in geringem Ausmaß möglich, eine Kontraindikation gegen die Impfung ergibt sich jedoch wegen des geringen Schweregrades dieser Formen der Exazerbationen und der potenziellen Schutzwirkung der Impfung vor schweren Infektionskomplikationen in keinem Fall. 
Systemische Nebenwirkungen treten nach der Anwendung der Influenzavakzine nur in der Größenordnung des Plazeboeffektes auf.

Die Influenzavakzine reduziert bei Personen >65 Jahre die Gesamtsterblichkeitsrate während der Influenzasaison signifikant.

\section{Antigen-Drift: Influenza-Viren sind genetisch sehr labil. Daher entstehen ständig neue Varianten mit mäßig veränderten Antigenen.}

Antigen-Shift: Es erfolgt eine Neuzusammensetzung
(„Reassortment“) ganzer Gensegmente. Dabei kommt
es zu gravierenden Mutationen der Oberflächen-Antigene
und der Virulenz. Die Viren sind hoch kontagiös
und virulent. Bei der Infektion treffen sie auf ein naives
humanes Immunsystem.

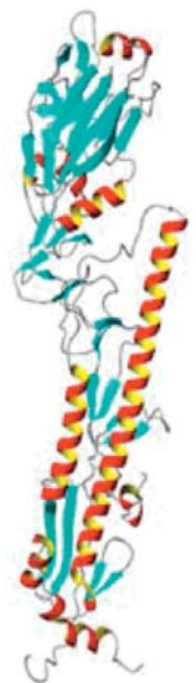

Abb. 2 Oberflächenantigene H und N (nach Nicholson KG, Wood JM, Zambon M. Influenza. Lancet 2003; 362: $1733-1745)$.

Die Spaltvakzine hat eine akzeptable Immunogenität und induziert wirksame Antikörpertiter bei $70-100 \%$ der geimpften gesunden Erwachsenen und immerhin noch bei $30-70 \%$ der Personen über 65 Jahren [4]. Auch bei niedrigen Dosen systemischer Steroide besteht ein Impfeffekt, der jedoch individuell geringer ausgeprägt sein kann. Eine niedrig dosierte Kortisontherapie ist keine Kontraindikation für die Impfung [5].

Bei Personen, die 65 Jahre oder jünger sind, wird ein 70 - 80\%-iger Schutz vor einer kulturellen bzw. serologisch nachgewiesenen Influenza-Infektion beschrieben (Abb.3). Bei Personen oberhalb des 65. Lebensjahres liegt der Schutzeffekt bei ca. 50\% [6]. Allerdings reduziert die Influenzavakzine auch in diesem Personenkreis die Gesamthospitalisierungsrate während der Influenzasaison signifikant [7]. Auch die Sterblichkeit von geimpften Personen oberhalb des 65. Lebensjahres wird durch die Spaltvakzine im

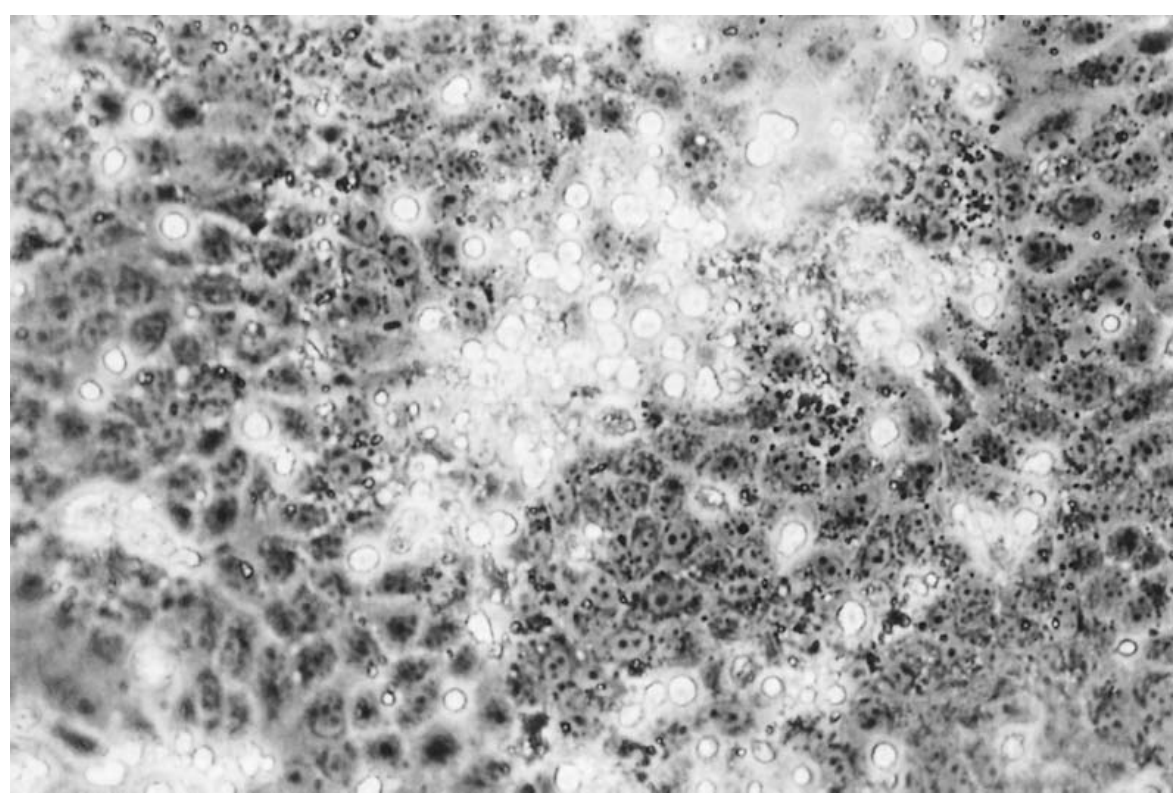

Abb. 3 Zytopathische Veränderungen durch eine Influenza-Infektion. 
Die biologische Wirkung der Influenzaspaltvakzine ist komplex.
Ganzen gesenkt [8]. Aus amerikanischen Quellen wissen wir, dass die Impfung von Personen im Alter von 65 und mehr Jahren als kosteneffektiv anzusehen ist [9]. Vermutlich gilt dies auch bei jüngeren Personen, wenn man die Kosten für krankheitsbedingtes Fernbleiben vom Arbeitsplatz mitberücksichtigt [10].

Die biologische Wirkung der Influenzaspaltvakzine ist komplex. Wie oben beschrieben, schützt die Vakzine die Mehrheit der geimpften Personen vor einer Influenzainfektion. Darüber hinaus wird jedoch auch die Gesamtmorbidität, insbesondere älterer Menschen hinsichtlich ambulant erworbener Pneumonie, und einer dekompensierten Herzinsuffizienz vermindert. In einer Untersuchung mit 1838 Personen, die älter als 60 Jahre waren, fand sich eine signifikante Protektion gegenüber einer serologisch nachgewiesenen Influenza-Infektion von 50\%, gegenüber einer klinisch diagnostizierten Influenza von $47 \%$ und gegenüber der Kombination aus serologisch und klinisch diagnostizierter Influenza von 58\% [11]. Eine Metaanalyse von 20 Kohorten-Studien zur Effektivität der Influenza-Schutzimpfung fand die folgenden Effektivitätsdaten: Verhinderung einer respiratorischen Erkrankung $=56 \%$; Verhinderung einer Pneumonie $=53 \%$; Verhinderung einer Hospitalisation $=50 \%$; Verhinderung eines Todesfalles $=68 \%$ [12]. Diese Daten wurden von neueren großen Kohorten-Studien bestätigt [13]. Daher ist die Indikation zur Grippeschutzimpfung breit ausgelegt [5].

\section{Wirkungen der Influenza-Schutzimpfung}

- Verminderung der serologisch und klinisch nachgewiesenen Infektionen

- Verminderung der respiratorischen Gesamtmorbidität

- Verminderung der Pneumonierate

- Verminderung der Krankenhauseinweisungen

- Verminderung der Gesamtmortalität

\section{Indikation zur Grippeschutzimpfung \\ (Ständige Impflkommission am Robert Koch-Institut 2004) \\ Standardimpfung: $S$ \\ Indikationsimpfung: I \\ Berufliches Risiko: B}

Jährliche Impfung mit einem von der WHO empfohlenen Impfstoff

S: Personen über 60 Jahre

I: Alle Personen mit erhöhter Gefährdung durch Grundleiden Chronische Atemwegserkrankungen einschließlich Asthma und COPD Chronische Krankheiten von Herz, Leber, Nieren Diabetes mellitus und andere Stoffwechselerkrankungen Multiple Sklerose (mit Infektions-getriggerten Schüben) Angeborene oder erworbene Immundefekte mit Rest-B- und /oder T-Zellfunktion HIV Infektion Bewohner von Alters- und Pflegeheimen

B: Medizinisches Personal, Mitarbeiter in Einrichtungen mit umfangreichem Publikumsverkehr, Personen, die als Infektionsquelle für von ihnen betreute ungeimpfte Risikopersonen fungieren können

I: Nach den Anweisungen der Gesundheitsbehörden, wenn in einer Epidemie der Impfstoff die relevante Virusvariante enthält. 
Zur Prävention der Influenza A-Infektion stehen die M-2-Inhibitoren Amantadin und Rimantadin und die Neuraminidase-Inhibitoren Zanamivir und Oseltamivir zur Verfügung.

Zanamivir und Oseltamivir sind auch gegen Influenza B-Infektionen wirksam.
Besondere Bedeutung kommt der Influenzaschutzwirkung unter dem Aspekt einer möglichen drohenden Pandemie durch ein Ressortment von Vogelgrippeviren und menschlichen Influenzaviren zu. Im Fall einer solchen Pandemie wäre die Schutzimpfung die wichtigste weltweit einsetzbare Strategie zur Eindämmung der Epidemie $[14,15]$.

\section{Medikamentöse Prävention der Influenza-Infektion}

Zur Prävention der Influenza A-Infektion stehen neben den M-2-Inhibitoren Amantadin und Rimantadin auch die Neuraminidase-Inhibitoren Zanamivir und Oseltamivir zur Verfügung, letztere sind auch gegen Influenza B-Infektionen wirksam. Sowohl in klinischen Studien als auch in systematischen Reviews konnte für alle Substanzen gezeigt werden, dass sie einen Präventionseffekt gegenüber der Influenza-Infektion haben $[16,17]$. Dies gilt vor allem im Fall eines unzureichenden Impfschutzes durch ein mismatching zwischen den aktuellen Influenzastämmen und den Impfstämmen als auch in einer Situation, bei der sich die Influenza in definierten Populationen, die nicht geimpft worden sind, ausbreitet (z.B. Seniorenheime).

\section{Medikamente zur Influenza-Prävention \\ M2-Inhibitoren \\ Rimantadin in Deutschland nicht verfügbar \\ Amantadinsulfat (Infex ${ }^{\circledR}$ u. a.) \\ Dosierung für Prävention und Therapie:}

Alter $<65$ Jahre $2 \times 100 \mathrm{mg} /$ Tag für 10 Tage

Alter $>65$ Jahre $1 \times 100 \mathrm{mg} /$ Tag für 10 Tage

UAW: QT-Zeitverlängerung (EKG-Kontrollen), Herzrhythmus-Störungen, Nierenfunktionsstörungen, Ödeme, Psychosen, Übelkeit, Mundtrockenheit, Überempfindlichkeitsreaktionen, Harnretention

Klinisch relevante Interaktionen: Multiple (insbesondere für QT-Zeitverlängerung: EKG-Kontrollen).

Kontraindikationen: Überempfindlichkeit, Schwangerschaft, Stillzeit, schwere Nierenfunktionsstörung, Engwinkelglaukom, Erregungszustände und Psychosen, gleichzeitige Therapie mit Kalium-sparenden Diuretika, schwere Prostata-Hyperplasie.

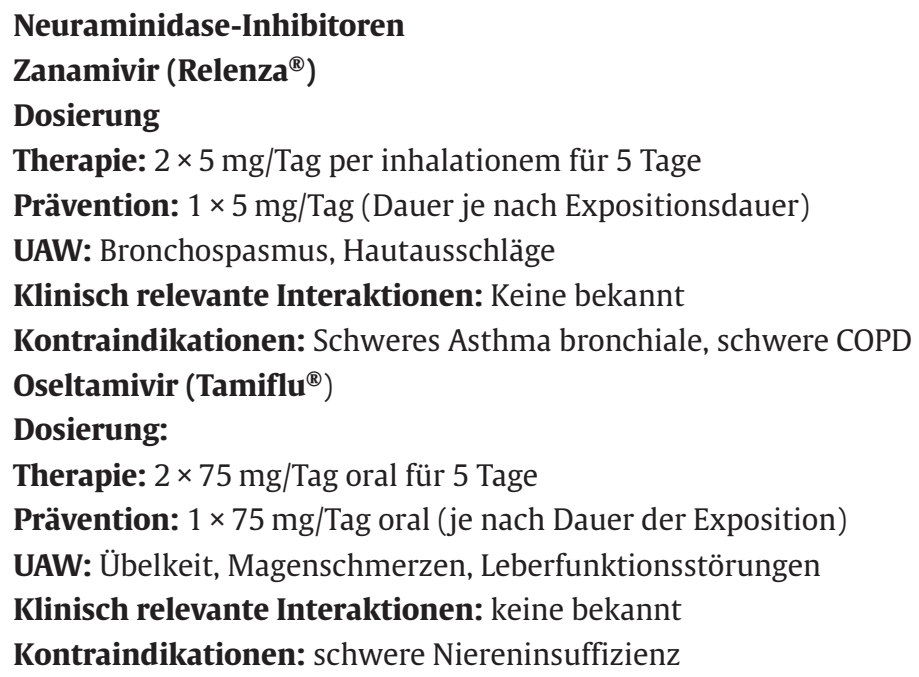


Der Einsatz der medikamentösen Grippe-Prävention sollte auf streng umschriebene Situationen wie z. B. einem Influenzaausbruch in einem Seniorenwohnheim mit niedriger Durchimpfungsrate beschränkt bleiben.

Bei einer Pandemie haben Neuraminidase-Inhibitoren unter Umständen eine große Bedeutung.

Die Schutzwirkung der Pneumokokken-Schutzimpfung gegenüber Pneumokokken-Erkrankungen ist nicht so gut belegt wie diejenige der Influenza-Schutzimpfung gegenüber der Grippe.

Die Pneumokokken-Schutzimpfung hat bei den Indikationsgruppen insgesamt eine mortalitätsverringernde Wirkung.

Obwohl der Impfstoff keine schleimhautgebundene Immunität gegenüber Pneumokokken-Infektionen vermitteln kann, besteht die Schutzwirkung gegenüber der Bakteriämie auch bei chronischer Bronchitis oder COPD.
Resistenzen von Influenza A- und B-Stämmen gegenüber allen zur Zeit verfügbaren antiviralen Substanzen sind beschrieben. Im Fall der Neuraminidase-Inhibitoren haben diese jedoch noch keine klinisch bedeutsame Relevanz [18].

Klar gesagt werden muss allerdings, dass die Indikationsstellung schwierig ist, da die Diagnose einer Influenza-Infektion klinisch gestellt werden muss [19]. Hilfreich können in diesem Zusammenhang aktuelle epidemiologische Daten sein, wie sie die verschiedenen Influenzanetzwerke liefern.

Kosten-/Nutzenstudien zum präventiven Einsatz der o.g. Medikamente liegen bisher nicht vor. Insofern sollte sich der Einsatz auf streng umschriebene Situationen wie z.B. einem Influenzaausbruch in einem Seniorenwohnheim mit niedriger Durchimpfungsrate beschränkt bleiben. Bei entsprechender Sensibilität eines Pandemie-Virus kommt insbesondere den Neuraminidase-Inhibitoren eine große Bedeutung zu [20].

\section{Pneumokokken-Schutzimpfung}

Der zur Zeit bei Erwachsenen am häufigsten eingesetzte Impfstoff ist die 23-valente Polysaccharidvakzine. Der Impfstoff wird intramuskulär verabreicht und kann, da es sich ebenfalls um einen Todimpfstoff handelt, parallel zur Grippeschutzimpfung verabreicht werden. Klinisch relevante systemische Impfreaktionen treten sehr selten auf. Schwerere Lokalreaktionen sind jedoch bei 2-3\% der Geimpften beschrieben [21] Leichte Lokalreaktionen mit Schwellung und leichten Schmerzen im Bereich der Impfstelle werden von 20-50\% der Geimpften berichtet. Der Impfstoff induziert den Anstieg spezifischer IgG-Antikörper in der Regel um den Faktor 2 [22]. Die Dauer der Schutzimpfung wird im Allgemeinen zwischen 3 und 10 Jahren angegeben, bei Patienten oberhalb des 70. Lebensjahres auch als lebenslang [5].

Problematisch ist die Schutzwirkung der Impfung gegenüber nachgewiesenen Pneumokokken-Erkrankungen. Bei Personen, die jünger als 55 Jahre sind, liegt die Schutzwirkung zwischen 70 und 80\%, gegenüber Pneumokokken-Pneumonien bei $40-70 \%$ und gegenüber dem Tod an Pneumonie an 30\% [23]. Bei Personen, die 65 Jahre und älter sind, nimmt man eine Schutzwirkung gegenüber nachgewiesenen PneumokokkenErkrankungen von 60-80\% an. Die Daten hinsichtlich der Schutzwirkung gegenüber Pneumokokken-Pneumonien sind hingegen sehr widersprüchlich. Insgesamt wird jedoch davon ausgegangen, dass bei allen geimpften Personen die PneumokokkenSchutzimpfung eine Verminderung der bakteriämisch verlaufenden PneumokokkenInfektionen erreicht. Hieraus resultiert auch die mortalitätsverringernde Wirkung des Impfstoffes. Da der Impfstoff keine schleimhautgebundene Immunität gegenüber Pneumokokken-Infektionen vermitteln kann, besteht keine Schutzwirkung gegenüber Atemwegsinfektionen i.S. einer Bronchitis oder akuten Exazerbation bei chronischer Bronchitis oder COPD [24].

\section{Wirkungen der Pneumokokken-Schutzimpfung \\ - Verminderung der bakteriämischen Infektionen \\ - Verminderung der Letalität}

Insgesamt muss man daher die Wirksamkeit der Pneumokokken-Schutzimpfung als deutlich schwächer einschätzen als diejenige der Influenza-Schutzimpfung. Das Robert Koch-Institut hat daher für die 23-valente Pneumokokken-Vakzine Empfehlungen herausgegeben [5], die den Personenkreis der zu Impfenden deutlich einschränken: Alter > 60 Jahre, angeborene oder erworbene Immundefekte mit T- und/oder B-zellulärer 
Restfunktion oder chronische Erkrankungen. Eine niedrig dosierte Kortisontherapie ist keine Kontraindikation für die Impfung [5].

\section{Indikation zur Pneumokokken-Schutzimpfung (ohne Kinder) (Ständige Impfkommission am Robert Koch-Institut 2004)

Impfung mit einem Polysaccharidimpfstoff, Wiederholung alle 6 Jahre

S: $\quad$ Personen über 60 Jahre

I: Alle Personen mit erhöhter Gefährdung durch Grundleiden

Chronische Atemwegserkrankungen einschließlich Asthma und COPD

Chronische Krankheiten von Herz, Leber, Nieren

Diabetes mellitus und andere Stoffwechselerkrankungen

Multiple Sklerose (mit Infektions-getriggerten Schüben)

Angeborene oder erworbene Immundefekte mit Rest-B- und/oder T-Zellfunk-

tion

HIV Infektion

Hypogammaglobulinämie, Komplement- oder Properdindefekte

Sichelzellanämie

Bei neoplastischen Erkrankungen

Nach Knochenmarkstransplantation

Funktionelle oder anatomische Asplenie

Liquorfistel

Vor Organtransplantation

Vor immunsuppressiver Therapie

\section{Antibiotika zur Prophylaxe bei chronischer Bronchitis und COPD}

Eine prophylaktische Antibiotikagabe bei Patienten mit chronischer Bronchitis oder COPD ist nicht effektiv.

Wegen unsicherer Wirkung und der erheblichen Kosten einer inhalativen Antibiotikaverabreichung wird eine solche bisher nicht empfohlen.
Patienten mit schwerer chronischer Bronchitis und COPD neigen zu mehrfach jährlichen Exazerbationen ihrer Erkrankung. Hierbei spielen primär Viren eine wichtige Rolle. Abb. 4. zeigt die zytopathischen Veränderungen durch eine Influenza-Infektion. Im Verlauf der Exazerbation kommt es jedoch bei einer Mehrzahl der Patienten zu einer bakteriellen Superinfektion. Um die rezidivierenden Infektionen des tiefen Atemwegstraktes zu vermeiden, hat man bereits in den Jahren 1960 - 1970 vor allem in England untersucht, ob die prophylaktische Gabe von gut verträglichen Antibiotika über die Herbst- und Wintersaison hilfreich sein kann [25]. Alle bisher verfügbaren Studien sind übereinstimmend zu dem Ergebnis gekommen, dass eine solche prophylaktische Antibiotikagabe nicht effektiv ist [26-28]. Insofern muss davon ausgegangen werden, dass die prophylaktische antibiotische Therapie von Patienten mit chronischer Bronchitis und COPD nicht empfohlen werden kann. Ausnahmen können sich ergeben, wenn es um Patienten mit schwerwiegender Bronchiektasen-Krankheit geht, allerdings ist die Evidenz für ein solches Vorgehen gering.

Die Frage, ob ähnlich wie bei der zystischen Fibrose inhalative Antibiotika [29] Patienten mit chronischer Bronchitis oder COPD vor bakteriellen akuten Exazerbationen ihrer Erkrankung schützen können, ist zur Zeit noch nicht eindeutig zu beantworten. Wegen der erheblichen Kosten, die mit einer inhalativen Antibiotikaverabreichung, z.B. von Aminoglykosiden einhergehen, kann aufgrund der bisher bekannten Datenlage ein solches Vorgehen nicht empfohlen werden. 
Der präventive Einsatz von Antibiotika bei Infekten des oberen Respirationstraktes verhindert tiefe Atemwegsinfektionen nicht.

Langwirksame $\beta_{2}$-Mimetika, Vagolytika und inhalative Steroide sind keine Präventionsstrategie gegenüber Infektionen des tiefen Respirationstraktes.

Physiotherapie senkt belegbar die Exazerbationsfrequenz nur bei zystischer Fibrose und Bronchiektasen.

Orale Mukolytika, die regelmäßig eingesetzt werden, haben einen mäßigen Effekt hinsichtlich der Verminderung der Exazerbationsfrequenz bei chronischer Bronchitis und chronisch-obstruktiver Atemwegserkrankung.

\section{Prävention von Infektionen des tiefen Respirationstraktes durch die frühzeitige antibiotische Therapie von Infekten des oberen Respirationstraktes}

Bisher existiert nur eine Studie bei Kindern, die geprüft hat, ob die sehr frühzeitig einsetzende Therapie einer oberen Atemwegsinfektion in der Lage ist, tiefe Atemwegsinfektionen und Pneumonien zu verhindern. Nach den Ergebnissen dieser Studie ergibt sich für den präventiven Einsatz einer solchen Therapie bei Kindern keine ausreichende Effektivitätsgrundlage. In einigen systematischen Reviews ist darüber hinaus untersucht worden, ob der Einsatz von Antibiotika oder antiviralen Substanzen bei akuter Rhinitis tiefe Atemwegsinfektionen verhindern kann. Alle diese Untersuchungen sind zu dem Schluss gekommen, dass ein solches therapeutisches Vorgehen ohne Effekt ist und daher nicht empfohlen werden kann.

\section{Langwirksame $\beta_{2}$-Mimetika, Vagolytika und inhalative Steroide als präventive Maßnahme gegenüber tiefen Atemwegsinfektionen bei Patienten mit COPD}

Obwohl einige Daten darauf hinweisen, dass unter einer regelmäßigen Therapie mit inhalativen Steroiden, langwirksamen Vagolytika und langwirksamen $\beta_{2}$-Mimetika die Exazerbationsfrequenz von Patienten mit schwerer chronisch-obstruktiver Atemwegserkrankung sinken kann, haben die bisher vorliegenden Studien nicht belegen können, dass diese nur in Subgruppen beobachtete Senkung der Exazerbationsfrequenz tatsächlich auf einer Senkung von Infektionen des unteren Respirationstraktes basiert [30]. Insofern ergeben sich unter dem Aspekt der Prävention von Atemwegsinfektionen und von Pneumonien zur Zeit keine Gründe für eine regelmäßige Therapie der Patienten mit diesen Substanzen.

\section{Prävention tiefer Atemwegsinfekte durch regelmäßige Physiotherapie}

Bei Patienten mit chronisch-bronchialer Hypersekretion und Bronchiektasen sowie mit zystischer Fibrose kann ein positiver Einfluss regelmäßig angewendeter physio-therapeutischer Maßnahmen auf die Exazerbationsfrequenz nachgewiesen werden [31,32]. Wiederum muss jedoch betont werden, dass diese Effekte nicht eindeutig auf eine Verminderung der Infektionsfrequenz zurückgeführt werden können. Nichtsdestotrotz bleibt bei den genannten Krankheitsbildern eine regelmäßige Drainage des tiefen Respirationstraktes Teil der Behandlungsstrategie [33].

\section{Orale Mukolytika zur Prävention tiefer Atemwegsinfektionen}

Neben einer Vielzahl kleinerer Studien liegt zumindest ein systemisches Review von insgesamt 23 Arbeiten vor, bei denen orale Mukolytika, wie z. B. Acetylcystein oder Ambroxol gegen Plazebo untersucht worden sind [34]. Die publizierten Daten zeigen in der Tat eine Reduktion der Exazerbationen um 0,84 pro Jahr. Dabei handelt es sich jedoch um Mischkollektive von Patienten mit chronisch-obstruktiver Atemwegserkrankung und einfacher chronischer Bronchitis. Insgesamt zeigen die Daten eine Reduktion der akuten Exazerbationen in den untersuchten Kollektiven um 29\%. Hieraus kann gefolgert werden, dass orale Mukolytika, die regelmäßig eingesetzt werden, einen mäßigen Effekt hinsichtlich der Verminderung der Exazerbationsfrequenz bei chronischer Bronchitis und chronisch-obstruktiver Atemwegserkrankung haben. Allerdings lässt sich aus diesen Daten nicht ableiten, dass bakterielle Atemwegsinfektionen bei den untersuchten Patientenkollektiven signifikant weniger auftreten. Da zusätzlich keine Aussa- 
Ein Effekt von Mukolytika auf bakterielle Atemwegsinfektionen wurde bisher nicht nachgewiesen.

Homöopathische Substanzen sind keine gesicherte präventive Strategie gegenüber tiefen Atemwegsinfektionen.

Die Haemophilus influenzae Vakzine zeigt keinen nachweislichen Effekt auf die Infektionsrate mit Haemophilus influenzae. ge gemacht werden kann, inwieweit die kontinuierliche Gabe oraler Mukolytika zu einer signifikanten Reduktion des Schweregrades von Exazerbationen bei Patienten mit chronischen Atemwegserkrankungen führt, sollte eine solche Therapie nicht generell durchgeführt werden, sondern in Einzelfällen in Betracht gezogen werden.

\section{Orale Mukolytika: \\ Acetylcystein}

Dosis: $600 \mathrm{mg} / \mathrm{Tag}$

UAW: Stomatitis, Erbrechen, Tinnitus, Kopfschmerzen, Überempfindlichkeitsreaktionen, Hemmung der Thrombozytenaggregation

Klinisch relevante Interaktionen: keine bekannt

Kontraindikationen: Überempfindlichkeit, Schwangerschaft, Stillzeit

Tagestherapiekosten: $0,30 €$

\section{Ambroxol}

Dosis: $30-60 \mathrm{mg} / \mathrm{Tag}$

UAW: Übelkeit, Sialorrhoe, Rhinorhoe, Obstipation, Dysurie, Mundtrockenheit, Überempfindlichkeitsreaktionen

Klinisch relevante Interaktionen: keine bekannt

Kontraindikationen: bronchiale Hypersekretion und gestörte Bronchomotorik (Abhustschwäche), Überempfindlichkeit, Schwangerschaft, Stillzeit, schwere Nieren- und Leberfunktionsstörung

Tagestherapiekosten: $0,15 €$

\section{Homöopathische Substanzen als präventive Strategie gegenüber tiefen Atemwegsinfektionen}

Eine ganze Anzahl von systematischen Reviews hat in den vergangenen Jahren untersucht, ob homöopathische Substanzen, wie z.B. die Echinacea-Derivate oder Zink u.a. eine präventive Strategie gegenüber tiefen Atemwegsinfektionen darstellen [35-39]. Obwohl hierzu eine ganze Anzahl von Studien vorliegt, sind alle systemischen Reviews zu der Schlussfolgerung gekommen, dass die untersuchten Substanzen keinen nachweisbaren Effekt haben. Insofern muss von ihrer Anwendung abgeraten werden.

\section{Orale Immunisationsstrategien mit Bakterienextrakten}

Zur Zeit werden auf diesem Gebiet zwei verschiedene präventive Strategien verfolgt.

Zum einen gibt es eine inaktivierte orale Haemophilus influenzae-Präparation, die als orale Vakzine eingesetzt wird, zum anderen steht ein inaktivierter Bakterienextrakt (OM 85 BV: S. pneumoniae, H. influenzae, Gram-negative Enterobacteriaceae u. a.) zur Verfügung. Die Analyse der vorliegenden Daten aus kontrollierten Studien in systematischen Reviews zeigt für die Haemophilus influenzae Vakzine eine signifikante Reduktion von akuten Exazerbationen bei Patienten mit chronischer Bronchitis in einem Zeitraum bis zu 6 Monaten nach einer entsprechenden oralen Gabe der Präparation [40]. Nach 9 und 12 Monaten lässt sich ein solcher Effekt nicht mehr nachweisen. Neben der Reduktion der Exazerbationsfrequenz zeigen die Daten ebenfalls eine Reduktion der Exazerbationsschwere. Insofern muss trotz der bedingten Datenlage (bisher lediglich 440 Studienpatienten) zum jetzigen Zeitpunkt davon ausgegangen werden, dass ein Effekt auf die Exazerbationsfrequenz existiert. Allerdings ergeben sich keine Hinweise auf eine echte Verringerung der Infektionsrate mit Haemophilus influenzae. In dieser Situation wird man weitere Untersuchung abwarten müssen, ehe eine generelle Empfehlung gegeben werden kann. 
OM 85 BV reduziert die Exazerbationsfrequenz um $17 \%$.

Bisher gibt es keinen Hinweis auf eine Verminderung der Infektionsfrequenz des tiefen Atemwegstraktes nach OM 85 BV Therapie.
Besser untersucht ist der orale Immunisationsstoff OM 85 BV. In einem aktuellen Review wurden die Daten von bisher 13 Studien mit insgesamt 1971 Patienten ausgewertet [41]. Hierbei ist jedoch zunächst festzustellen, dass lediglich zwei der 13 Studien mit zusammen 700 Patienten eine hohe Studienqualität hatten. Gefunden wurde eine Reduktion der Exazerbationsfrequenz um 17\%. Die Anzahl der, um eine Exazerbation zu vermeiden, zu behandelnden Patienten, lag bei 15,4. Deutlich war in den einzelnen Studien und in den systematischen Reviews auch ein Effekt auf die Symptomatik der Patienten, die nach einer oralen Immunisation mit der genannten Substanz über weniger exazerbationsbedingte Beschwerden klagten. Die wichtigsten beobachteten $\mathrm{Ne}-$ benwirkungen waren kutane Reaktionen und urologische Probleme, die bei 3,3 bzw. $8 \%$ der mit dem Bakterienextrakt behandelten Patienten auftraten.

Die Autoren des systematischen Reviews gehen nach ihrer Analyse davon aus, dass die Bakterienextrakte in der Lage sind, die Symptomatik von Patienten mit chronischer Bronchitis und COPD zu bessern. Allerdings ergibt sich nach ihrer Analyse keine Evidenz, dass tatsächlich in signifikantem Ausmaß Exazerbationen vermieden werden können. Insbesondere ergibt sich kein Hinweis auf eine Verminderung der Infektionsfrequenz des tiefen Atemwegstraktes.

Aussagen zur Kosteneffizienz liegen nicht vor. Berücksichtigt man die oben dargestellten Daten, so kann eine Empfehlung zum Einsatz oral-immunisierender Substanzen zum jetzigen Zeitpunkt nicht ausgesprochen werden.

\section{Fazit für die Praxis}

- Die wichtigste Präventionsmaßnahme gegen respiratorische Infektionen ist die Grippeschutzimpfung. Die Indikation zu dieser Impfung wird in Deutschland breit gestellt.

- Antiviral wirksame Medikamente haben in der Praxis nur eine eingeschränkte Bedeutung für bestimmte Risikokonstellationen.

- Die Pneumokokken-Schutzimpfung ist weniger wirksam als die Influenza-Schutzimpfung. Sie schützt jedoch Risikopatienten vor bakteriämischen Verläufen und senkt damit die Letalität von schweren Pneumokokken-Infektionen.

- Antibiotika, Mukolytika, Bronchospasmolytika, inhalative Steroide, physikalische Therapieverfahren und orale Immunisationsverfahren haben keine gesicherte Bedeutung für die Prävention von Infektionen der tiefen Atmungsorgane. 
1 Bridges CB, Winquist AG, Fukuda Ket al. Advisory Committee on Immunization Practies: Prevention and control of influenza. MMWR Recomm Rep 2000; 49: 1- 38

2 Beyer WE, de Bruijn IA, Palache AMet al. Protection against influenza after annually repeated vaccination: a meta-analysis of serologic and field studies. Arch Intern Med 1999; 159 (2): $182-188$

3 Demicheli V, Rivetti D, Deeks JJet al. Vaccines for preventing influenza in healthy adults. Cochrane Database Syst Rev 2001; (4): CD001269

4 Gross PA, Quinnan Jr GV, Weksler MEet al. Immunization of elderly people with high doses of influenza vaccine. J Am Geriatr Soc 1988; 36 (3): 209-212

5 STIKO. Empfehlungen der Ständigen Impfkommission (STIKO) am Robert Koch-Institut/Stand. Epidemiol Bull 2004; 30: 235-250

6 Bernstein E, Kaye D, Abrutyn Eet al. Immune response to influenza vaccination in a large healthy elderly population. Vaccine 1999; 17 (1): 82 - 94

7 Nichol K. Improving influenza vaccination rates for high-risk inpatients. Am J Med 1991; 91 : 584-588

8 Nichol KL, Margolis KL, Wouremna Jet al. Effectiveness of influenza vaccine in the elderly. Gerontology 1996; 42 (5): 274-279

9 Nichol KL, Margolis KL, Wuorenma Jet al. The efficacy and cost effectiveness of vaccination against influenza among elderly persons living in the community. N Engl J Med 1994; 331 (12): $778-784$

${ }^{10} \mathrm{Nichol} \mathrm{KL}$. Cost-benefit analysis of a strategy to vaccinate healthy working adults against influenza. Arch Intern Med 2001; 161 (5): 749-759

${ }^{11}$ Govaert TM, Sprenger MJ, Dinant GJet al. Immune response to influenza vaccination of elderly people. A randomized double-blind placebo-controlled trial. Vaccine 1994; 12 (13): $1185-1189$

${ }^{12}$ Gross PA, Hermogenes AW, Sacks HSet al. The efficacy of influenza vaccine in elderly persons. A meta-analysis and review of the literature. Ann Intern Med 1995; 123 (7): 518 - 527

${ }^{13}$ Nichol KL, Baken L, Nelson A. Relation between influenza vaccination and outpatients visits, hospitalization, and mortality in elderly persons with chronic lung disease. Ann Intern Med 1999; 130 (5): $397-403$

${ }^{14}$ Laver G, Garman E. Pandemic influenza: its origin and control. Microbes Infect 2002; 4 (13): $1309-1316$

${ }^{15}$ Tam JS. Influenza A (H5N1) in Hong Kong: an overview. Vaccine 2002; 20 Suppl 2: 77-81

${ }^{16}$ Jefferson TO, Demicheli V, Deeks JJet al. Amantadine and rimantadine for preventing and treating influenza A in adults. Cochrane Database Syst Rev 2002; (3): CD001169

${ }^{17}$ Jefferson T, Demicheli V, Deeks Jet al. Neuraminidase inhibitors for preventing and treating influenza in healthy adults. Cochrane Database Syst Rev 2000; (2): CD001265

${ }^{18}$ Boivin G, Goyette N. Susceptibility of recent Canadian influenza A and B virus isolates to different neuraminidase inhibitors. Antiviral Res 2002; 54 (3): 143-147

${ }^{19}$ Sintchenko V, Gilbert GL, Coiera Eet al. Treat or test first? Decision analysis of empirical antiviral treatment of influenza virus infection versus treatment based on rapid test results. J Clin Virol 2002; 25 (1): 15-21

${ }^{20}$ Oxford JS, Bossuyt S, Balasingam Set al. Treatment of epidemic and pandemic influenza with neuraminidase and M2 proton channel inhibitors 696. Clin Microbiol Infect 2003; 9 (1): 1 - 14

${ }^{21}$ Schaberg T. Pneumokokken-Schutzimpfung. Pneumologie 2001; 55 (12): 543 - 545

${ }^{22}$ Hedlund JU, Kalin ME, Ortqvist ABet al. Antibody response to pneumococcal vaccine in middle-aged and elderly patients recently treated for pneumonia. Arch Intern Med 1994; 154 (17): $1961-1965$

${ }^{23}$ Cornu C, Yzebe D, Leophonte Pet al. Efficacy of pneumococcal polysaccharide vaccine in immunocompetent adults: a meta-analysis of randomized trials. Vaccine 2001; 19 (32): $4780-4790$

${ }^{24}$ Nichol KL, Baken L, Wuorenma Jet al. The health and economic benefits associated with pneumococcal vaccination of elderly persons with chronic lung disease. Arch Intern Med 1999; 159 (20): $2437-2442$

${ }^{25}$ Medical Research Council. Value of chemoprophylaxis and chemotherapy in early chronic bronchitis. A report to the Medical Research Council by their working party on trials of chemotherapy in early chronic bronchitis. Br Med J 1966; 5499: 1317 - 1322

${ }^{26}$ Johnston RN, McNeill RS, Smith DHet al. Five-year winter chemoprophylaxis for chronic bronchitis. Br Med J 1969; 4 (678): 265 - 269

${ }^{27}$ Mars G, Santarato R, Necchi D, Silva A. Prolonged minimal dose administration of sulphamethopyrazine in the treatment and prophylaxis of infectious diseases in the old. Panminerva Med 1997; 9: 607-610

${ }^{28}$ Black P, Staykova T, Chacko Eet al. Prophylactic antibiotic therapy for chronic bronchitis 112 . Cochrane Database Syst Rev 2003; (1): CD004105

${ }^{29}$ Ramsey B, Pepe MS, Quan JMet al. Intermittent administration of inhaled tobramycin in patients with cystic fibrosis. N Engl J Med 1999; 340: 23 - 30

${ }^{30}$ The COPD Guidelines Group of the Standards of Care Committee of the BTS. BTS guidelines for the management of chronic obstructive pulmonary disease. Thorax 1997; 52: $1-28$

${ }^{31}$ Bradley J, Moran F, Greenstone M. Physical training for bronchiectasis. Cochrane Database Syst Rev 2002; (3): CD002166

32 Varekojis SM, Douce FH, Flucke RLet al. A comparison of the therapeutic effectiveness of and preference for postural drainage and percussion, intrapulmonary percussive ventilation, and 
high-frequency chest wall compression in hospitalized cystic fibrosis patients. Respir Care 2003; 48 (1): $24-28$

${ }^{33}$ Jones A, Rowe BH. Bronchopulmonary hygiene physical therapy in bronchiectasis and chronic obstructive pulmonary disease: a systematic review. Heart Lung 2000; 29 (2): 125-135

${ }^{34}$ Poole PJ, Black PN. Mucolytic agents for chronic bronchitis or chronic obstructive pulmonary disease. Cochrane Database Syst Rev 2003; (2): CD001287

${ }^{35}$ Melchart D, Linde K, Fischer Pet al. Echinacea for preventing and treating the common cold. Cochrane Database Syst Rev 2000; (2): CD000530

${ }^{36}$ Belongia EA, Berg R, Liu K. A randomized trial of zinc nasal spray for the treatment of upper respiratory illness in adults. Am J Med 2001; 111 (2): $103-108$

${ }^{37}$ Vickers AJ, Smith C. Homoeopathic Oscillococcinum for preventing and treating influenza and influenza-like syndromes. Cochrane Database Syst Rev 2000; (2): CD001957

${ }^{38}$ Marshall I. Zinc for the common cold. Cochrane Database Syst Rev 2000; (2): CD001364

${ }^{39}$ Jackson JL, Lesho E, Peterson C. Zinc and the common cold: a meta-analysis revisited. J Nutr 2000; 130 (5S Suppl): $1512-1515$

${ }^{40}$ Foxwell AR, Cripps AW. Haemophilus influenzae oral vaccination against acute bronchitis. Cochrane Database Syst Rev 2003; (2): CD001958

${ }^{41}$ Steurer-Stey C, Bachmann LM, Steurer Jet al. Oral purified bacterial extracts in chronic bronchitis and COPD: a systematic review. Chest 2004; 126: 1645-1655

\section{Professor Dr. Tom Schaberg, FCCP}

Zentrum für Pneumologie · Diakoniekrankenhaus Rotenburg

Verdener Str. 200

27356 Rotenburg

E-mail: schaberg@diako-online.de 
Welche Antwort trifft zu?
Welche Antwort ist
zutreffend?

Was trifft zu?

A Pro Jahr treten in der Bundesrepublik Deutschland mehr als 8 Millionen akute Bronchitiden auf.

B Pro Jahr treten in der Bundesrepublik Deutschland zirka 800.000 ambulant erworbene Pneumonien auf.

C Pro Jahr treten in der Bundesrepublik Deutschland zirka 4 Millionen akute Exazerbationen der chronischen Bronchitis und chronisch obstruktiven Atemwegserkrankung auf.

D a und $b$ sind richtig

E a-c sind richtig

Welche Antwort ist richtig?
A Der Influenzaimpfstoff ist eine Lebendvakzine.

B Die Influenzavakzine wird subkutan appliziert.

C Die Influenzavakzine wird intramuskulär appliziert.

D Eine Allergie gegen Hühnereiweiß ist keine Kontraindikation für die Grippeschutzimpfung.

E Systemische Nebenwirkungen treten bei 10-20\% der Geimpften auf.

A Die Grippeschutzimpfung schützt in jedem Fall vor einer Influenzainfektion.

B Der Schutzeffekt der Grippeschutzimpfung beträgt bei Personen $>65$ Jahre mindestens $80 \%$.

C Die Grippeschutzimpfung ist bei Personen $>65$ Jahre nicht kosteneffektiv.

D Die Grippeschutzimpfung reduziert bei Personen $>65$ Jahre die Gesamtsterblichkeit während der Influenzasaison.

E Die Grippeschutzimpfung hat keinen Einfluss auf die Hospitalisierungsraten von Personen >65 Jahren.

A Zur Prävention eine Influenza B-Infektion eignen sich vor allem Amantadin und Rimantadin.

B Gegenüber den verfügbaren antiviralen Substanzen resistente Influenzavirus-Stämme sind nicht bekannt.

C Antivirale Medikamente mit Wirksamkeit gegen Influenzaviren sind eine sinnvolle Alternative zur Grippeschutzimpfung.

D Im Fall eine Pandemie können Neuraminidase-Inhibitoren eine wichtige Rolle spielen.

E Bei Verdacht auf eine Influenza-Infektion sollten antiviral wirksame Medikamente breit eingesetzt werden.

A Die Schutzwirkung der Influenza- und Pneumokokken-Schutzimpfung ist gleich.

B Der Pneumokokken-Impfstoff umfasst Polysaccharide von 22 verschiedenen PneumokokkenSerotypen.

C Die Pneumokokken-Schutzimpfung schützt vor Pneumokokken-bedingten akuten Bronchitiden.

D Personen, die älter als 70 Jahre sind, müssen wegen der verminderten Antikörperbildung jährlich geimpft werden.

E Die COPD ist eine Indikation für die Pneumokokken-Schutzimpfung. 

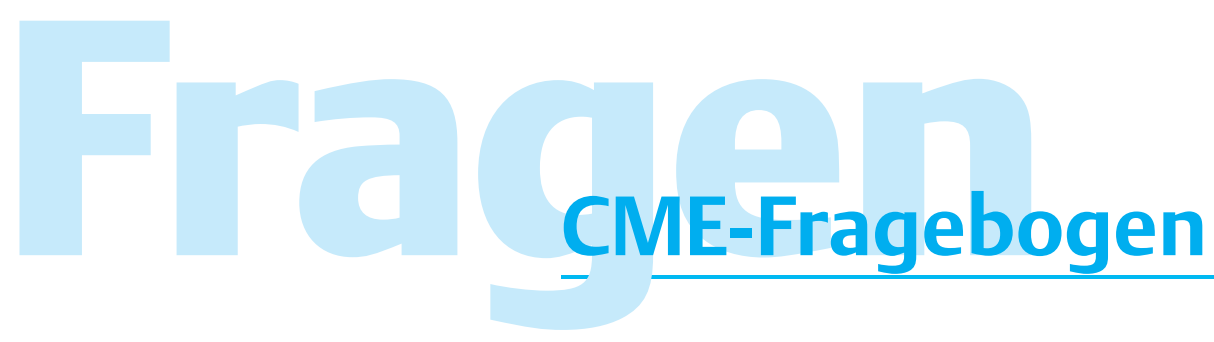

6 Was trifft zu? Welche Aussage ist
richtig?

\footnotetext{
Was trifft zu?
}

Was trifft nicht zu?

10

Welche Antwort ist richtig?
A Die prophylaktische Gabe von breitwirksamen Antibiotika schützt COPD-Patienten vor akuten Exazerbationen ihrer Erkrankung.

B Bei schwerwiegender Bronchiektasen-Krankheit kann die langfristige präventive Gabe von Antibiotika gerechtfertigt sein.

C Die Inhalation von Aminoglykosiden ist bei COPD-Patienten eine kosteneffektive Maßnahme.

D a und c sind richtig

E Alle Aussagen sind falsch.

A Inhalative Steroide haben keinen Einfluss auf die Exazerbationsfrequenz bei Patienten mit schwerer COPD.

B Langwirksame $\beta_{2}$-Mimetika und Vagolytika verhindern rezidivierende Infekte der tiefen Atemwege bei Patienten mit COPD.

C Unter dem Aspekt der Prävention von bakteriellen tiefen Atemwegsinfektionen besteht eine klare Indikation für inhalative Steroide bei Patienten mit schwerer COPD.

D Regelmäßige Physiotherapie senkt die Infektionsrate bei COPD-Patienten.

E Die regelmäßige Drainage des Bronchialsystems senkt die Exazerbationsfrequenz bei Patienten mit zystischer Fibrose.

A Mukolytika haben keinen Effekt auf die Exazerbationsfrequenz von Patienten mit COPD.

B Alle Patienten mit chronischer Bronchitis und COPD sollten orale Mukolytika erhalten.

C Mukolytika schützen vor bakteriellen Infektionen des Respirationstraktes.

D Der Effekt von oralen Mukolytika auf die Exazerbationsfrequenz von COPD-Patienten liegt in der Größenordnung des Plazebo-Effektes.

E a-d sind nicht richtig.

A Die orale Immunisation mit Bakterienextrakten ist eine kosteneffiziente Maßnahme.

B OM 85BV hat keinen Effekt auf die Exazerbationsfrequenz von Patienten mit chronischer Bronchitis und COPD.

C Nebenwirkungen von OM 85BV sind bisher nicht beschrieben.

D Die orale Immunisation mit Bakterienextrakten wird zur Prävention tiefer Atemwegsinfektionen breit empfohlen.

E $a-d$

A Alle homöopathische Substanzen sind eine wirkungsvolle Präventionsstrategie gegenüber tiefen Atemwegsinfektionen.

B Nur Echinacea-Derivate sind wirkungsvoll.

C Nur Zink ist wirkungsvoll.

D Homöopathische Substanzen können Infektionen der unteren Atemwege nicht verhindern.

E $\mathrm{a}-\mathrm{d}$ sind falsch. 
Bitte kopieren, ausfüllen und an die unten angegebene Adresse senden.

\section{Angaben zur Person}

Evaluation
Name, Vorname, akad. Titel:

Straße, Hausnummer: PLZ/Ort.

Ich bin Mitglied der Ärztekammer:

Jahr meiner Approbation:

Ich befinde mich in der Weiterbildung zum:

Ich habe eine abgeschlossene Weiterbildung in/für:

seit/Jahr der Facharztanerkennung:

Spezialisierung innerhalb des Fachgebiets: $\square$ nein $\quad \square$ ja, welche?

Ich möchte folgende Zusatzbezeichnungen erwerben:

Ich habe folgende Zusatzbezeichnungen: seit:

Ich bin tätig als:

$\square$ Assistenzarzt $\square$ Oberarzt $\square$ Chefarzt in folgender Klinik:

$\square$ Niedergelassener Arzt, seit $\square$ im Ballungsraum $\square$ im ländlichen Raum

$\square$ Sonstiges (bitte eintragen):

Führen Sie in Ihrer Praxis diagnostische und therapeutische Auftragsleistungen im Bereich des Fortbildungsthemas durch?

$\square$ nein $\square$ ja, welche?

Bieten Sie in der Diagnostik und Therapie im Bereich des Fortbildungsthemas Besonderheiten in Ihrer Praxis/Klinik an?

$\square$ nein $\square$ ja, welche?

Machen Sie diese ggf. als Praxisbesonderheiten geltend?

$\square$ nein $\square$ ja, welche?

1. Wie viele Patienten diagnostizieren/behandeln Sie im Zusammenhang mit dem Fortbildungsthema pro Jahr?

2. Bitte benennen Sie die 3 wichtigsten für Sie noch offenen Probleme in der Diagnostik/Therapie des Fortbildungsthemas:

3. Bezüglich der Diagnostik/Therapie im Rahmen des Fortbildungsthemas $\square$ fühle ich mich nach Studium des Beitrages in meiner Strategie bestätigt. $\square$ hat sich meine Strategie folgendermaßen verändert - bitte benennen:

4. Wurden aus der Sicht Ihrer täglichen Praxis wichtige Aspekte des Themas
a) außer Acht gelassen?
$\square$ nein $\square$ ja, welche?
b) zu knapp abgehandelt?
$\square$ nein $\square$ ja, welche?
c) überbewertet?
$\square$ nein
ja, welche? 


\begin{tabular}{l} 
Ihr Ergebnis \\
(wird vom Verlag ausgefüllt) \\
Sie haben___ von___ Fragen \\
richtig beantwortet und somit \\
$\square$ bestanden und 3 Punkte erworben. \\
$\square$ nicht bestanden. \\
Stuttgart, \\
\hline (Stempel/Unterschrift)
\end{tabular}

\section{Lernerfolgskontrolle}

Antworten zum Quiz

(nur eine Antwort pro Frage ankreuzen)
5. Etwa wieviel Prozent des Beitrages haben Ihnen
a) zur Auffrischung bereits bekannten Wissens gedient:
$\square<10 \% \quad \square<25 \% \quad \square<50 \% \quad \square \geq 50 \%$
b) zur Erweiterung Ihres Spezialwissens gedient:
$\square<10 \% \quad \square<25 \% \quad \square<50 \% \quad \square \geq 50 \%$

6. Ergeben sich für Sie aus wirtschaftlichen Gründen Limitierungen im Einsatz von im Beitrag genannten Diagnose-/Therapieverfahren?

$\square$ nein $\square$ ja, welche?

7. Stehen Ihnen aus logistischen Gründen Limitierungen im Einsatz von im Beitrag genannten Diagnose-/Therapieverfahren nicht/nur eingeschränkt zur Verfügung?

$\square$ nein $\square$ ja, welche?

8. Die Fragen lassen sich

$\square$ aus dem Studium des Beitrages allein beantworten.

$\square$ nur unter Zuhilfenahme zusätzlicher Literatur beantworten.

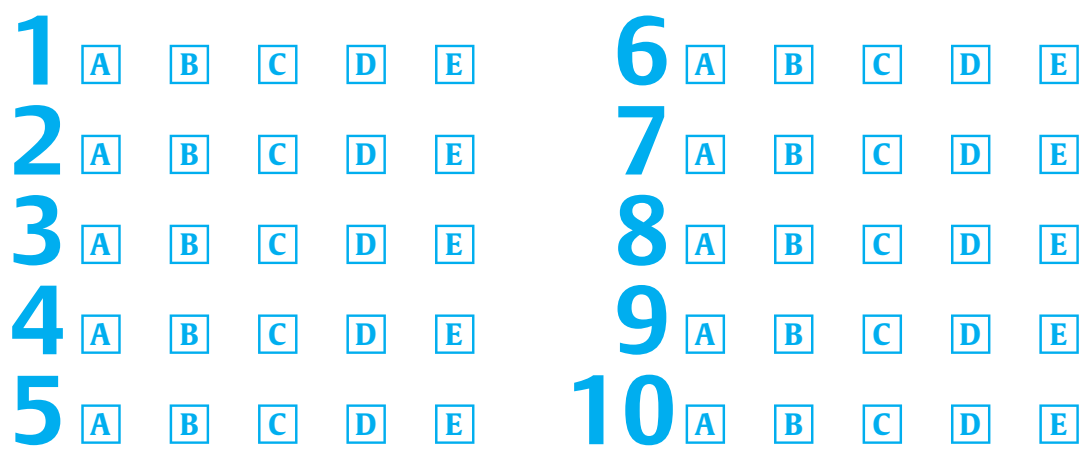

\section{Erklärung}

Ich versichere, dass ich die Beantwortung der Fragen selbst und ohne fremde Hilfe durchgeführt habe.

Ort/Datum

Unterschrift

Nichtabonnenten bitte hier CME-Wertmarke aufkleben bzw. Abonnentennummer eintragen
Bitte senden Sie den vollständig ausgefüllten Antwortbogen und einen an Sie selbst adressierten und ausreichend frankierten Rückumschlag an den Georg Thieme Verlag KG, CME, Joachim Ortleb, Postfach 301120, 70451 Stuttgart. Einsendeschluss ist der 31. März 2006 (Datum des Poststempels). Die Zertifikate werden spätestens 14 Tage nach Erhalt des Antwortbogens versandt. Von telefonischen Anfragen bitten wir abzusehen.

\section{Teilnahmebedingungen der zertifizierten Fortbildung}

Für diese Fortbildungseinheit erhalten Sie 1 Fortbildungspunkt im Rahmen des Fortbildungszertifikates der Ärztekammern. Hierfür

- müssen $80 \%$ Fragen richtig beantwortet sein.

- muss die oben stehende Erklärung vollständig ausgefüllt sein. Unvollständig ausgefüllte Bögen können nicht berücksichtigt werden!

- muss Ihre Abonnentennummer im entsprechenden Feld des Antwortbogens angegeben oder eine CME-Wertmarke im dafür vorgesehenen Feld aufgeklebt sein.

\section{CME-Wertmarken für Nichtabonnenten}

CME-Wertmarken (für Teilnehmer, die die „Pneumologie“ nicht abonniert haben) können beim Verlag zu folgenden Bedingun- gen erworben werden: 6er-Pack Thieme-CME-Wertmarken, Preis $€$ 63,- inkl. MwSt., Artikel-Nr. 901916; 12er-Pack Thieme-CMEWertmarken, Preis € 99,- inkl. MwSt., Artikel-Nr. 901917. Bitte richten Sie Bestellungen an: Georg Thieme Verlag KG, Kundenservice, Postfach 301120, 70451 Stuttgart.

\section{Wichtige Hinweise}

Die CME-Beiträge der „Pneumologie“ wurden durch die Ärztekammer Nordrhein anerkannt. Die „Pneumologie“ ist zur Vergabe der Fortbildungspunkte für diese Fortbildungseinheit berechtigt. Diese Fortbildungspunkte werden von anderen zertifizierenden Ärztekammern anerkannt. Die Vergabe der Fortbildungspunkte ist nicht an ein Abonnement gekoppelt! 